\title{
Cosmological Parameters From the Quad Cmb Polarization Experiment
}

\section{Citation}

Castro, P. G., P. Ade, J. Bock, M. Bowden, M. L. Brown, G. Cahill, S. Church, et al. 2009. "Cosmological Parameters From the Quad Cmb Polarization Experiment." The Astrophysical Journal 701 (2) (July 28): 857-864. doi:10.1088/0004-637x/701/2/857.

\section{Published Version}

doi:10.1088/0004-637X/701/2/857

\section{Permanent link}

http://nrs.harvard.edu/urn-3:HUL.InstRepos:25757767

\section{Terms of Use}

This article was downloaded from Harvard University's DASH repository, and is made available under the terms and conditions applicable to Other Posted Material, as set forth at http:// nrs.harvard.edu/urn-3:HUL.InstRepos:dash.current.terms-of-use\#LAA

\section{Share Your Story}

The Harvard community has made this article openly available.

Please share how this access benefits you. Submit a story.

\section{Accessibility}




\title{
COSMOLOGICAL PARAMETERS FROM THE QUAD CMB POLARIZATION EXPERIMENT
}

\author{
P. G. Castro ${ }^{1,10}$, P. Ade ${ }^{2}$, J. Bock ${ }^{3,4}$, M. Bowden ${ }^{2,5}$, M. L. Brown ${ }^{1,11}$, G. Cahill ${ }^{6}$, S. Church $^{5}$, T. Culverhouse ${ }^{7}$,

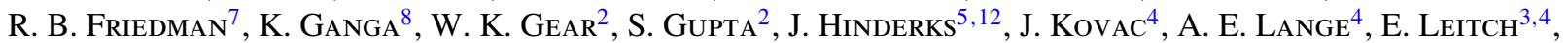 \\ S. J. Melhuish ${ }^{9}$, Y. Memari ${ }^{1}$, J. A. Murphy ${ }^{6}$, A. Orlando ${ }^{2,4}$, C. Pryke ${ }^{7}$, R. Schwarz ${ }^{7}$, C. O’Sullivan ${ }^{6}$, L. Piccirillo ${ }^{9}$,

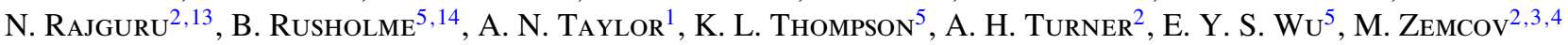 \\ (THE QUAD COLLABORATION) \\ ${ }^{1}$ Scottish Universities Physics Alliance (SUPA), Institute for Astronomy, University of Edinburgh, Royal Observatory, Blackford Hill, Edinburgh EH9 3HJ, UK \\ ${ }^{2}$ School of Physics and Astronomy, Cardiff University, Queen's Buildings, The Parade, Cardiff CF24 3AA, UK \\ 3 Jet Propulsion Laboratory, 4800 Oak Grove Dr., Pasadena, CA 91109, USA \\ ${ }^{4}$ California Institute of Technology, Pasadena, CA 91125, USA \\ ${ }^{5}$ Kavli Institute for Particle Astrophysics and Cosmology and Department of Physics, Stanford University, 382 Via Pueblo Mall, Stanford, CA 94305, USA \\ ${ }^{6}$ Department of Experimental Physics, National University of Ireland Maynooth, Maynooth, Co. Kildare, Ireland \\ ${ }^{7}$ Kavli Institute for Cosmological Physics, Department of Astronomy \& Astrophysics, Enrico Fermi Institute, University of Chicago, 5640 South Ellis Avenue, \\ Chicago, IL 60637, USA \\ ${ }^{8}$ APC UMR 7164 (Univ. Paris Diderot-Paris 7 - CNRS - CEA - Obs. de Paris), 10, rue Alice Domon et Léonie Duquet, 75205 Paris Cedex 13, France \\ ${ }^{9}$ School of Physics and Astronomy, University of Manchester, Manchester M13 9PL, UK \\ Received 2008 December 19; accepted 2009 May 11; published 2009 July 28
}

\begin{abstract}
In this paper, we present a parameter estimation analysis of the polarization and temperature power spectra from the second and third season of observations with the QUaD experiment. QUaD has for the first time detected multiple acoustic peaks in the $E$-mode polarization spectrum with high significance. Although QUaD-only parameter constraints are not competitive with previous results for the standard six-parameter $\Lambda \mathrm{CDM}$ cosmology, they do allow meaningful polarization-only parameter analyses for the first time. In a standard six-parameter $\Lambda \mathrm{CDM}$ analysis, we find the QUaD TT power spectrum to be in good agreement with previous results. However, the QUaD polarization data show some tension with $\Lambda \mathrm{CDM}$. The origin of this $1 \sigma-2 \sigma$ tension remains unclear, and may point to new physics, residual systematics, or simple random chance. We also combine QUaD with the five-year WMAP data set and the SDSS luminous red galaxies 4th data release power spectrum, and extend our analysis to constrain individual isocurvature mode fractions, constraining cold dark matter density, $\alpha_{\mathrm{cdmi}}<0.11$ (95\% confidence limit $(\mathrm{CL})$ ), neutrino density, $\alpha_{\mathrm{ndi}}<0.26$ (95\% CL), and neutrino velocity, $\alpha_{\mathrm{nvi}}<0.23$ (95\% CL), modes. Our analysis sets a benchmark for future polarization experiments.
\end{abstract}

Key words: cosmic microwave background - cosmological parameters - methods: statistical - polarization

Online-only material: color figures

\section{INTRODUCTION}

The anisotropies of the cosmic microwave background (CMB) radiation are among the most important tests of cosmology. The large-angle Sachs-Wolfe effect, multiple acoustic oscillations, and the Silk damping tail in the temperature power spectrum have now been confirmed by a range of experiments from the largest angular scales down to angular scales of a few arcminutes (Dunkley et al. 2009; Reichardt et al. 2009). The full repository of CMB data available, in conjunction with other cosmological observables, such as data coming from the largescale distribution of galaxies or supernova type Ia observations, are extremely well described by the spatially flat $\Lambda \mathrm{CDM}$ cosmological model.

A generic prediction of cosmology is that the CMB photons should be polarized at the $10 \%$ level. The polarization field

\footnotetext{
${ }^{10}$ Current address: CENTRA, Departamento de Física, Edifício Ciência, Instituto Superior Técnico, Av. Rovisco Pais 1, 1049-001 Lisboa, Portugal.

${ }^{11}$ Current address: Cavendish Laboratory, University of Cambridge, J. J. Thomson Avenue, Cambridge CB3 OHE, UK.

${ }^{12}$ Current address: NASA Goddard Space Flight Center, 8800 Greenbelt Road, Greenbelt, Maryland 20771, USA.

${ }^{13}$ Current address: Department of Physics and Astronomy, University College London, Gower Street, London WC1E 6BT, UK.

${ }^{14}$ Current address: Infrared Processing and Analysis Center, California

Institute of Technology, Pasadena, CA 91125, USA.
}

can be decomposed into two components: primary even-parity, curl-free $E$-modes are generated at the last scattering surface by both scalar and tensor metric perturbations (gravitational waves); primary odd-parity $B$-modes are generated only by tensor perturbations due to gravitational waves passing through the primordial plasma. Secondary anisotropies in both the $E$ - and $B$-mode polarizations arise at the epoch of reionization, while $E$ - and $B$-modes are mixed by gravitational lensing by intervening large-scale structure along the line of sight (see, e.g., Hu \& White 1997). Observations of this linearly polarized component provide an important consistency check of the standard model and a detection of primordial gravitational waves in the oddparity $B$-mode on large angular scales would be strong evidence for inflation.

After the DASI ${ }^{15}$ experiment (Kovac et al. 2002) made the first measurement of $E$-mode power, other experiments have provided us with further measurements at a wide range of angular scales (Barkats et al. 2005; Readhead et al. 2004; Montroy et al. 2006; Sievers et al. 2007; Page et al. 2003; Bischoff et al. 2008; Nolta et al. 2009). Despite this we were still lacking precision measurements of its power spectra down to arcminute scales, as we have for the temperature. The $B$-mode polarization has not yet been detected and only upper limits have been determined.

15 DASI stands for "Degree Angular Scale Interferometer." 
The $\mathrm{QUaD}^{16}$ experiment is at the forefront of this smallscale polarization quest, and after three years of observations has delivered the highest resolution $E$-mode spectrum and the tightest upper limits on the $B$-modes yet measured. This is a significant improvement over the first season of data results, previously reported by Ade et al. (2008). In particular, the sensitivity of QUaD has allowed us to see, for the first time, four acoustic oscillations in the $E$-mode spectrum and all significant oscillations in the $T E$ spectrum to $\ell=2000$. The overall consistency of peak phases and spacings between the temperature and QUaD EE data was shown in Pryke et al. (2009), hereafter referred to as the "Power Spectra Paper".

In this paper, we concentrate on using the QUaD temperature and polarization power spectra to constrain the standard cosmological model. Using this baseline model, we analyze the different contributions coming from each of the QUaD spectra. We also go beyond the standard $\Lambda \mathrm{CDM}$ model using the QUaD data in combination with WMAP and SDSS to constrain an isocurvature contribution.

\section{COSMOLOGICAL PARAMETER ESTIMATION: METHODOLOGY}

\subsection{Monte Carlo Markov Chain}

The Monte Carlo Markov Chain (MCMC) method is a method designed to efficiently explore an unknown probability distribution function (PDF) by sequentially drawing samples from it according to a proposal probability function, in our case the Metropolis algorithm (Metropolis et al. 1953 among others). The ensemble of these samples constitutes a Markov Chain whose distribution corresponds to that of the unknown PDF. We adopted the Gelman and Rubin $R$-statistic to verify that our chains are properly mixed and converged (Gelman \& Rubin 1992; Verde et al. 2003), and compare sets of four chains of around 100,000 steps from which we remove a burn-in period during which our criterium is not met. We use at least 80,000 steps after burn-in.

The rate of convergence of the Markov Chain is slowed down by degeneracies between parameters, and the choice of the step size in the Metropolis algorithm. Therefore, we apply a standard partial reparameterization of the parameter space as suggested in Kosowsky et al. (2002). To further reduce the remaining degeneracies between parameters, we apply a change of basis in parameter space described in Tegmark et al. (2004) which uses a covariance matrix to take account of all the correlations between parameters. In the new basis, the new parameters have zero average and unit variance.

When we have a fair sample of the underlying distribution, the MCMC method trivializes marginalization to a simple projection of the points of the chain. The mean marginalized value of each parameter will hereafter be called "the mean recovered model." Note that some authors refer to the mean recovered model as the "best-fit model."

To obtain constraints on the mean parameters values, one simply produces the one-dimensional histograms of the chain values for each parameter, and calculate confidence intervals using the $p^{\text {th }}$ and $\left(1-p^{\text {th }}\right)$ quantiles of the histograms as in Verde et al. (2003). Normally, we use $68 \%$ equivalent to a nominal $1 \sigma$ constraint. However, if the constraint as defined above hits the

16 QUaD stands for "QUEST and DASI." In turn, QUEST is "Q \& U Extragalactic Survey Telescope." The two experiments merged to become QUaD in 2003. prior boundary on one end we instead choose the level which contains $95 \%$ of the total probability and quote an upper (or lower) limit. We shall also plot two-dimensional marginalized parameter distributions with $68 \%$ and $95 \%$ contours estimated at $\Delta \ln L=-2.3$ and -6.17 from the peak values. Assuming a Gaussian distribution, we quote $\chi^{2}$ values corresponding to our mean recovered model, and the Probability To Exceed (PTE), $P\left(>\chi^{2} \mid v\right)$, which gives the random probability to have found the measured value of $\chi^{2}$ or greater by chance, for $v$ degrees of freedom.

\subsection{The Likelihood and Nuisance Marginalization}

The likelihood for the measured $C_{\ell}$ band powers is well approximated by a Gaussian distribution, given by

$$
P\left(\hat{C}_{b} \mid C_{b}^{\mathrm{th}}\right) \propto \exp \left[-\frac{1}{2} \Delta C_{b}(p) M_{b b^{\prime}}^{-1} \Delta C_{b^{\prime}}^{\dagger}(p)\right],
$$

where $\Delta C_{b}(p)=\hat{C}_{b}-C_{b}^{\text {th }}(p), \hat{C}_{b}$ are the measured QUaD binned $C_{\ell}$ band powers, $C_{b}^{\text {th }}(p)$ are the theoretical power spectra which depend on the cosmological parameters, $p$, and which have been transformed to predictions of the binned spectra by means of the experimental band-power window function (BPWF), and $M_{b b^{\prime}}=\left\langle\Delta C_{b} \Delta C_{b^{\prime}}^{\dagger}\right\rangle$ is the measured $\hat{C}_{b}$ bandpower covariance matrix (BPCM).

The BPCM is estimated from an ensemble of simulations of the CMB sky, assuming a fixed fiducial $\Lambda \mathrm{CDM}$, run through the QUaD analysis pipeline (see, e.g., Brown et al. 2005). In our analysis, this BPCM remains independent of the cosmological parameters, but in principle we should vary it as we move around parameter space. This rescaling of the sample variance with $C^{\text {th }}$ is equivalent to saying that $P\left(C_{b}^{\text {th }}(p) \mid \hat{C}_{b}\right)$ is $\log$ normally distributed, and in the case where noise is present, offset log-normally distributed. Bond et al. (1998) propose a transformation of the band powers and BPCM which accounts for this effect and allows use of a fixed BPCM. We have tested the impact of this transformation on our full data set and find that the difference in parameter estimates and uncertainties is insignificant.

The Gaussian likelihood has the added benefit that we can simplify the marginalization over beam and calibration nuisance parameters. Indeed, in this case, one can directly apply an analytic marginalization scheme as in Bridle et al. (2002). The resulting marginalization results in extra terms added to the BPCM of our Gaussian likelihood that acts as a source of extra noise. The likelihood function is then given by

$$
\ln L=-\frac{1}{2} \Delta C_{b} M_{b b^{\prime}}^{\prime-1} \Delta C_{b^{\prime}}^{\dagger}-\frac{1}{2} \operatorname{Tr} \ln M^{\prime}
$$

where

$$
M_{b b^{\prime}}^{\prime}=M_{b b^{\prime}}+\sigma_{\mathrm{Cal}}^{2} C_{b} C_{b^{\prime}}^{\dagger}+2 \sigma_{b}^{2} \delta \ell_{b}^{2} C_{b} \ell_{b^{\prime}}^{2} C_{b^{\prime}}^{\dagger}
$$

is the marginalized BPCM, where $\sigma_{\mathrm{Cal}}^{2}$ is the variance on the calibration, $\sigma_{b}=\theta_{\mathrm{FWHM}} / \sqrt{8 \ln 2}, \theta_{\mathrm{FWHM}}$ is the effective beam size, $\delta$ is the fractional beam error, and $\ell_{b}$ is the average multipole in a bin.

In assessing the goodness of fit of our mean recovered models, and when comparing the measured $\hat{C}_{b}$ band powers with the WMAP5 $\Lambda$ CDM model, we shall use the $\chi^{2}$-statistic, introduced previously in Section 2.1, and which we define by

$$
\chi^{2}=\Delta C_{b} M_{b b^{\prime}}^{\prime-1} \Delta C_{b^{\prime}}^{\dagger},
$$


Table 1

Basic Cosmological Mean Parameter Constraints Using QUaD Band-Power Spectra for Various Data Combinations

\begin{tabular}{|c|c|c|c|c|c|c|}
\hline Symbol & Q08 TT/TE/EE/BB & Q08 TE/EE/BB & Q08 TT & Q08 TE & Q08 $E E / B B$ & WMAP5 \\
\hline$\Omega_{b} h^{2}$ & $0.0334_{-0.0040}^{+0.0039}$ & $0.0319 \pm 0.0046$ & $0.0242_{-0.0057}^{+0.0058}$ & $0.0398 \pm 0.0051$ & $0.0366_{-0.0161}^{+0.0159}$ & $0.02261{ }_{-0.00063}^{+0.00062}$ \\
\hline$\Omega_{m} h^{2}$ & $0.119_{-0.015}^{+0.014}$ & $0.117 \pm 0.017$ & $0.133_{-0.033}^{+0.035}$ & $0.149 \pm 0.025$ & $0.155_{-0.035}^{+0.034}$ & $0.1329 \stackrel{+0.0064}{-0.0065}$ \\
\hline$h$ & $0.91 \pm 0.09$ & $0.90 \pm 0.10$ & $0.75_{-0.18}^{+0.17}$ & $0.87 \pm 0.11$ & $0.77 \pm 0.18$ & $0.717_{-0.027}^{+0.026}$ \\
\hline$A_{s} e^{-2 \tau a}$ & $0.66 \pm 0.08$ & $0.63 \pm 0.09$ & $0.64_{-0.10}^{+0.09}$ & $0.63 \pm 0.13$ & $0.79 \pm 0.21$ & $0.614_{-0.018}^{+0.017}$ \\
\hline$n_{s}^{a}$ & $0.809 \pm 0.078$ & $0.766 \pm 0.152$ & $0.848_{-0.121}^{+0.117}$ & $1.337_{-0.254}^{+0.259}$ & $0.534_{-0.161}^{+0.155}$ & $0.967 \pm 0.015$ \\
\hline $100 \theta$ & $1.035 \pm 0.006$ & $1.034 \pm 0.007$ & $1.034 \pm 0.015$ & $1.035 \pm 0.007$ & $1.024 \pm 0.014$ & $1.040 \pm 0.003$ \\
\hline$\chi^{2}(v)^{b}$ & $88.60(86)$ & $74.78(63)$ & $12.73(17)$ & $19.67(17)$ & $33.16(40)$ & \\
\hline PTE: $P\left(\geqslant \chi^{2} \mid v\right)$ & $40.26 \%$ & $14.72 \%$ & $75.38 \%$ & $29.14 \%$ & $76.94 \%$ & \\
\hline$\chi^{2}{\text { (WMAP5 } \mid \mathrm{Q} 08)^{c}}^{c}$ & $108.63(92)$ & $86.99(69)$ & $14.48(23)$ & $31.44(23)$ & $41.62(46)$ & \\
\hline PTE(WMAP5|Q08) & $11.36 \%$ & $7.07 \%$ & $91.24 \%$ & $11.24 \%$ & $65.60 \%$ & \\
\hline
\end{tabular}

Notes.

a The pivot point for $A_{s}$ and $n_{s}$ is $k_{p}=0.05 \mathrm{Mpc}^{-1}$ for both the QUaD data and WMAP5 data.

b $\chi^{2}$ for the six-parameter mean recovered model against QUaD data, with the number of degrees of freedom in brackets.

c $\chi^{2}$ for WMAP5 mean recovered model given the QUaD data set, with the number of degrees of freedom in brackets.

where we use the nuisance marginalized $\mathrm{BPCM}, M^{\prime}{ }_{b b^{\prime}}$, as defined in the previous equation.

\subsection{The Standard Cosmological Model}

We parameterize our flat $\Lambda \mathrm{CDM}$ cosmological model with the following standard set of six cosmological parameters: the Hubble constant, $H_{0}=100 h \mathrm{~km} \mathrm{~s}^{-1} \mathrm{Mpc}^{-1}$; the physical matter density, $\Omega_{m} h^{2}$; the physical baryon density, $\Omega_{b} h^{2}$; the amplitude of scalar fluctuations, $A_{s}$, related to the curvature fluctuations by $\Delta_{R}(k)=2.95 .10^{-9} A_{s}(k)$; the scalar spectral index, $n_{s}$; and the optical depth, $\tau$. When using QUaD data by itself, we present the combination $A_{s} e^{-2 \tau}$ as our individual constraints on the degenerate parameters $A_{s}$ and $\tau$ are prior driven and thus biased, as explained in the Appendix. Initial conditions are taken to be purely adiabatic with an initial powerlaw mass-density perturbation spectrum. Due to the range of angular scales probed by QUaD, the pivot point we use when analyzing QUaD data by itself is $k_{p}=0.05 \mathrm{Mpc}^{-1}$ (note this is independent of $h$ ). When comparing our QUaD results with $W M A P$, we regenerate $W M A P$ best-fit values based on this pivot value using our own pipeline, however, when adding $\mathrm{QUaD}$ data to other data sets for a combined analysis we revert to the WMAP preferred pivot scale of $k_{p}=0.002 \mathrm{Mpc}^{-1}$.

To generate our theoretical spectra, we use the publicly available CAMB code (Lewis et al. 2000), including the effects of reionization, and gravitational lensing by foreground structure. We impose the following flat priors in the likelihood analysis: $0 \leqslant \Omega_{c} h^{2} \leqslant 1,0 \leqslant \Omega_{b} h^{2} \leqslant 1,0.005 \leqslant \theta \leqslant 0.1,0 \leqslant \tau \leqslant 0.8$, $0 \leqslant A_{s} \leqslant 2.5$, and $0 \leqslant n_{s} \leqslant 2 . \Omega_{c} h^{2}$ is the physical cold dark matter density and the parameter $\theta$ is the angular sound horizon at decoupling, defined as $\theta=r_{s}\left(a_{*}\right) / D_{A}\left(a_{*}\right)$, where $a_{*}$ is the scale factor at decoupling, $r_{s}\left(a_{*}\right)$ is the physical size of the sound horizon at decoupling, and $D_{A}\left(a_{*}\right)$ is the angular diameter distance to the surface of last scattering. Note that the partial reparameterization of the parameter space as suggested in Kosowsky et al. (2002) introduces an implicit prior on the $h$ parameter.

\section{RESULTS: BASIC FIVE-PARAMETER CONSTRAINTS}

\subsection{QUaD-Only Constraints}

The QUaD data set we use to constrain cosmological parameters are the optimally combined spectra obtained from the
$100 \mathrm{GHz}, 150 \mathrm{GHz}$ and frequency-cross temperature and polarization $E$ - and $B$-power spectra, measured in 23 band powers over angular multipoles from $200<\ell<2000$ as described in the Power Spectra Paper. We estimate a $10 \%$ uncertainty on both the calibration (in power units) and the beam sizes, assuming an effective beam of 4.1 arcmin. ${ }^{17}$

In parameter estimation, we use the diagonal and the first two off-diagonal terms of the BPCM for TT-TT, TE-TE, EE-EE, and $B B-B B$ covariances, but only the diagonal and first off-diagonal terms in the $T T-T E$ and $T E-E E$ covariances. This is motivated by the need to avoid excessive noise in the off-diagonal terms of the BPCM, due to its estimation from numerical simulations. We also ignore the covariance between $T T$ and $E E$, which is much smaller than the other terms.

We explore various sets of combinations of the QUaD temperature and polarization data in order to understand the new information each spectrum brings to parameter estimation. In Table 1, we present the mean recovered models. Figure 1 shows the corresponding two-dimensional marginalized contour projections of the likelihood in the five-parameter space.

All of the statistics shown verify that the QUaD TT temperature power spectrum is compatible with the results from WMAP5. This is a nontrivial test, since the overlap of scales measured by QUaD and WMAP5 is only in the range $\ell \approx 200$ to $\ell \approx 950$, while the QUaD data extends to $\ell \approx 2000$ with good signal to noise.

However, the analysis involving the polarization spectra is in less good agreement, yielding a high value of the baryon content. Indeed for the $T E / E E / B B$ combination, we have $\Omega_{b} h^{2}=$ $0.0319 \pm 0.0046$, compared to $\Omega_{b} h^{2}=0.02261 \pm 0.00062$ from WMAP5. The $\chi^{2}$ of the WMAP5 best-fit model for this data set has a PTE of $7 \%$ indicating a modest degree of tension. The spectrum responsible for this behavior seems to be the $T E$ spectrum.

Clearly, the $T E$ only constraints are weak and most parameters are prior driven, but surprisingly we obtain constraints on $\Omega_{b} h^{2}$ and $\Omega_{m} h^{2}$ that are not influenced by their choice of priors. Figure 2 shows the two-dimensional projected likelihood surface for the $\left(\Omega_{b} h^{2}, \Omega_{m} h^{2}\right)$ parameter space. To illustrate the difference with the $T T$ only contours, we overplot them. In addition, we show the results from WMAP5 and the big bang nucleosynthesis (BBN) constraint of $\Omega_{b} h^{2}=0.0214 \pm 0.002$

$\overline{17}$ The data set is publicly available online at http://quad.uchicago.edu/quad. 


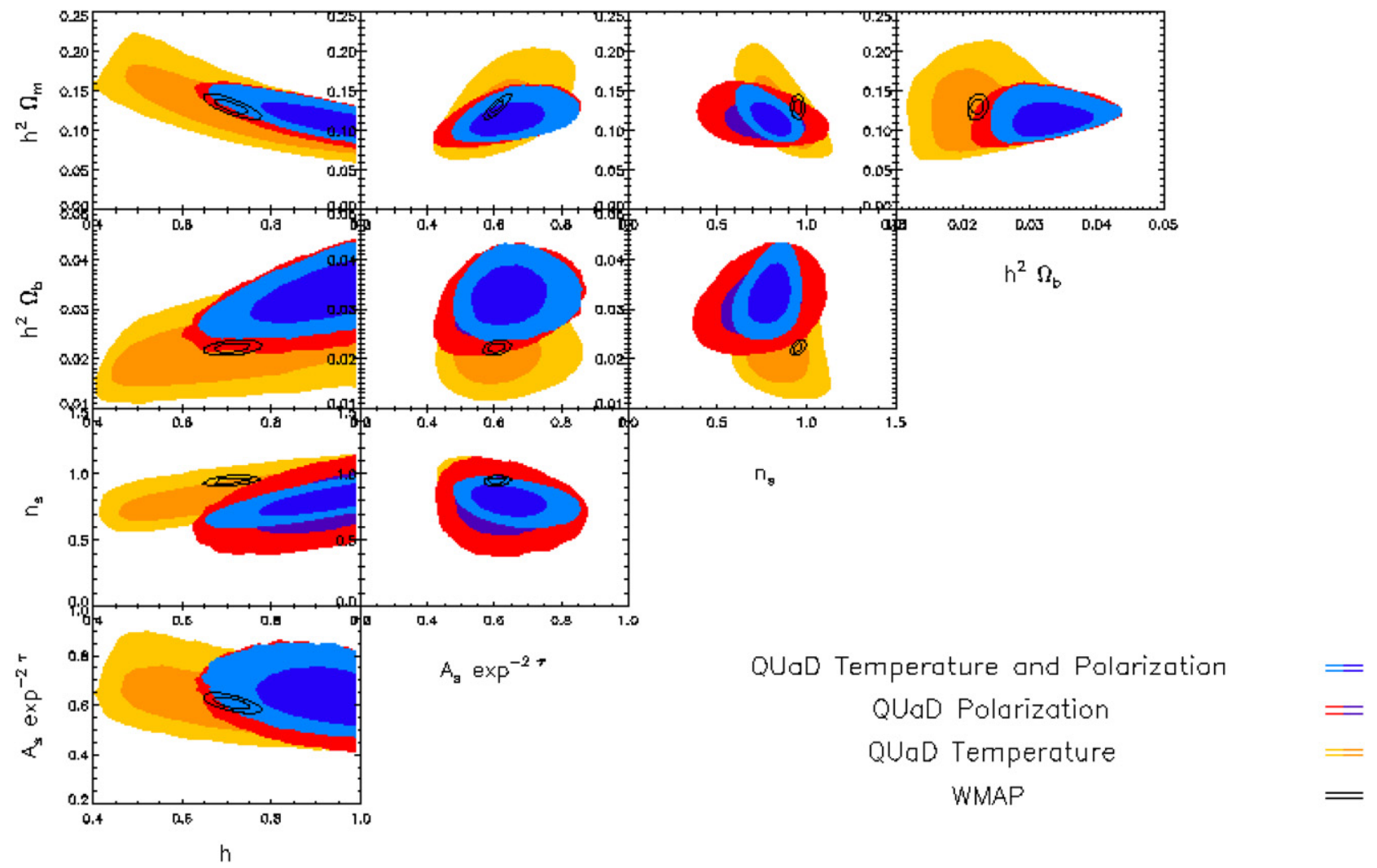

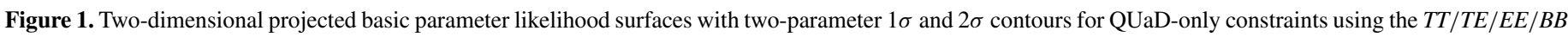

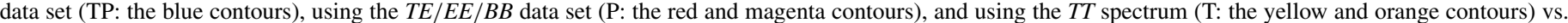
the WMAP5 constraints (black/empty contours). Pivot scale used is $k_{p}=0.05 \mathrm{Mpc}^{-1}$.

(A color version of this figure is available in the online journal.)

from Kirkman et al. (2003). We also show in Figure 3 the $T E$ QUaD band-power spectrum versus its mean recovered model and the WMAP5 full data set best-fit model. This figure visually illustrates the differences of the mean recovered models between the two data sets in terms of height and location of the peaks. The main reason for the higher baryon density parameter seems to be larger acoustic oscillations at higher multipole in $T E$, as well as a shift to higher multipoles of the peaks, resulting in a slight degeneracy with $h$, which may explain its high value. The origin of this source of tension is unclear, but could be due to a new physical mechanism, residual systematics or random chance.

Another interesting result comes from the $E E$ and $B B$ spectra. As expected they provide very little information on parameters, and the $\chi^{2}$ of the WMAP5 best-fit model for this data set has an acceptable PTE of $65.6 \%$. They do, however, have an unusual feature; the preferred range of scalar spectral index values is low $\left(n_{s}=0.534_{-0.161}^{+0.155}\right)$, showing nevertheless a big tail to high $n_{s}$. So although $T T$ and $T E$ share the majority of the constraining power, the $E E$ and $B B$ spectra exert an influence in combination with the remaining spectra by restricting the $n_{s}$-range to low values.

If we combine all the spectra together then the polarization data dominate the constraints. The majority of parameters are consistent with the WMAP5 results, but the spectral index $n_{s}$ is lower, influenced by the $E E / B B$ contribution, and the Hubble parameter $h$ and $\Omega_{b} h^{2}$ are higher, driven by the polarization data, in particular the TE spectrum. Compared to the BBN value $\Omega_{b} h^{2}$ is almost $3 \sigma$ away. Figure 4 shows a comparison between our $T T / T E / E E / B B$ data and models and the WMAP5 data and

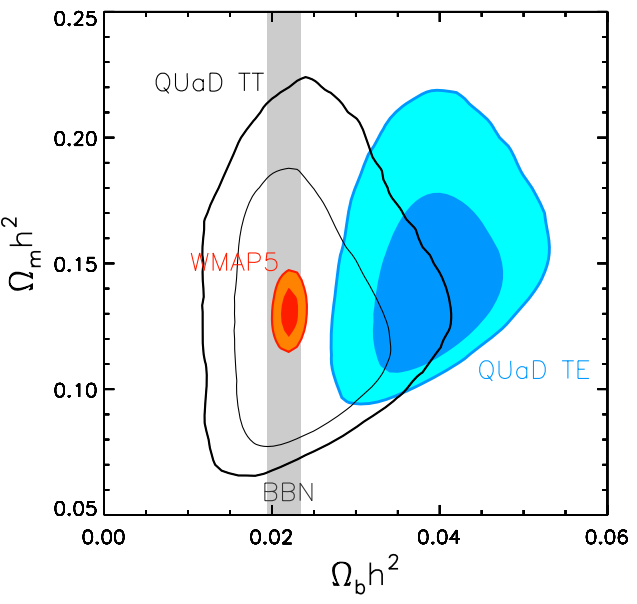

Figure 2. Two-dimensional marginalized contours of the parameters $\Omega_{b} h^{2}$ vs. $\Omega_{m} h^{2}$ obtained from QUaD TE data only. Also plotted are the contours from QUaD TT data only, the results from WMAP5, and the BBN constraint (Kirkman et al. 2003).

(A color version of this figure is available in the online journal.)

best-fit model. The $\chi^{2}$ of the WMAP5 best-fit model indicates that there is an $11.36 \%$ chance that the combined QUaD spectra are a realization of this model.

\subsection{Combining QUaD with Other Data Sets}

In this section, we will add to the QUaD spectra the WMAP five-year $T T, T E, E E$, and $B B$ data set. We use the WMAP5 


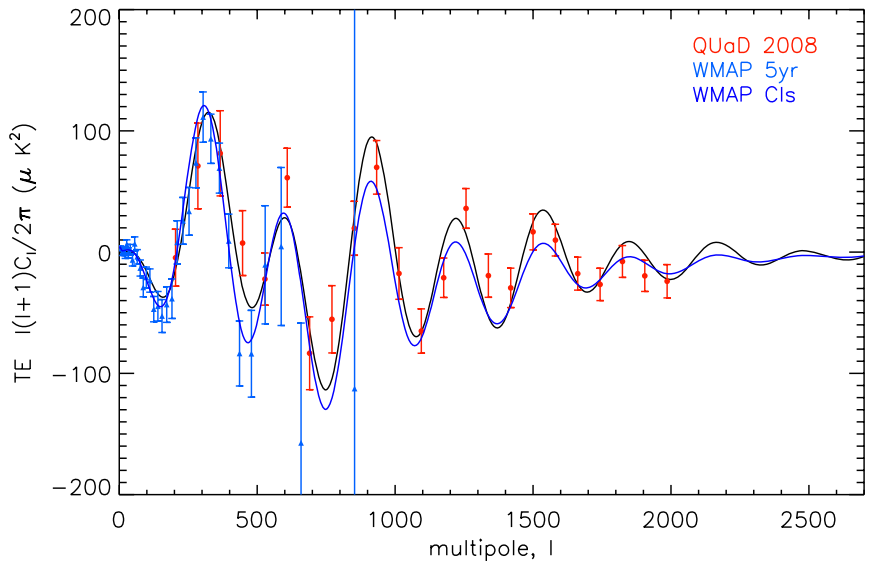

Figure 3. QUaD TE data band-power spectrum (in red) vs. the QUaD TE mean recovered model (in solid black) together with WMAP5 best-fit model (blue). For the TE mean recovered model, we assumed the WMAP5 best-fit value for the optical depth ( $\tau=0.087$ ), which corresponds to an amplitude of $A_{s}=0.75$, given our $A_{s} e^{-2 \tau}$ constraint.

(A color version of this figure is available in the online journal.)

likelihood code, publicly available on the LAMBDA Web site, ${ }^{18}$ and their methodology (Dunkley et al. 2009), but do not include the Sunyaev Zel'dovich (SZ) marginalization. We shall assume that the two data sets are independent, as the QUaD data only cover a small fraction of the WMAP5 sky, and the overlap in multipole range is only partial.

We will further add large-scale structure data from the SDSS luminous red galaxies (LRGs) fourth data release using publicly available likelihood code, measurements and window functions (Tegmark et al. 2006). Results from the SDSS LRG and the main SDSS galaxy samples are consistent, but the former provides higher signal-to-noise ratio. We use the SDSS LRG matter power spectrum over wavenumbers $k_{\mathrm{SDSS}}<0.07 \mathrm{~h} \mathrm{Mpc}^{-1}$ so that we do not have to consider any nonlinear correction. We marginalize over the amplitude of the galaxy power spectrum which removes any dependence on the galaxy bias parameter, $b_{g}$, and linear redshift-space distortion.

As can be seen in Table 2, the QUaD TT/TE/EE/BB power spectra have little impact on the baseline six-parameter mean parameter fit when combined with WMAP5. This is perhaps unsurprising, given the accuracy of the WMAP5 measurement of the first acoustic peak in the TT spectrum, and its low- $\ell$ power in $T T$ and $T E$. The impact it does have is to tighten the error bars on parameters determined from the relative heights of acoustic peaks, i.e., on the baryon density, $\Omega_{b} h^{2}$, and the matter density, $\Omega_{m} h^{2}$, as QUaD data add a substantial amount of well-defined peak information at high- $\ell$.

When we combine the SDSS LRG and WMAP5 data with the QUaD data, we see an improvement compared to the QUaD and WMAP5 combination, as expected. This improvement is mostly due to the extra constraining power on $\Omega_{m} h^{2}$ and $\Omega_{b} h^{2}$ coming from the break scale in the SDSS galaxy power spectrum, and the baryon acoustic oscillations. However, the QUaD data still reduce the uncertainty on $\Omega_{b} h^{2}$.

\section{BEYOND THE STANDARD SIX-PARAMETER MODEL: ISOCURVATURE MODES}

Theoretical predictions of isocurvature modes and their evolution, and the role of CMB polarization observations in

\footnotetext{
18 LAMBDA Web site: http://lambda.gsfc.nasa.gov/.
}
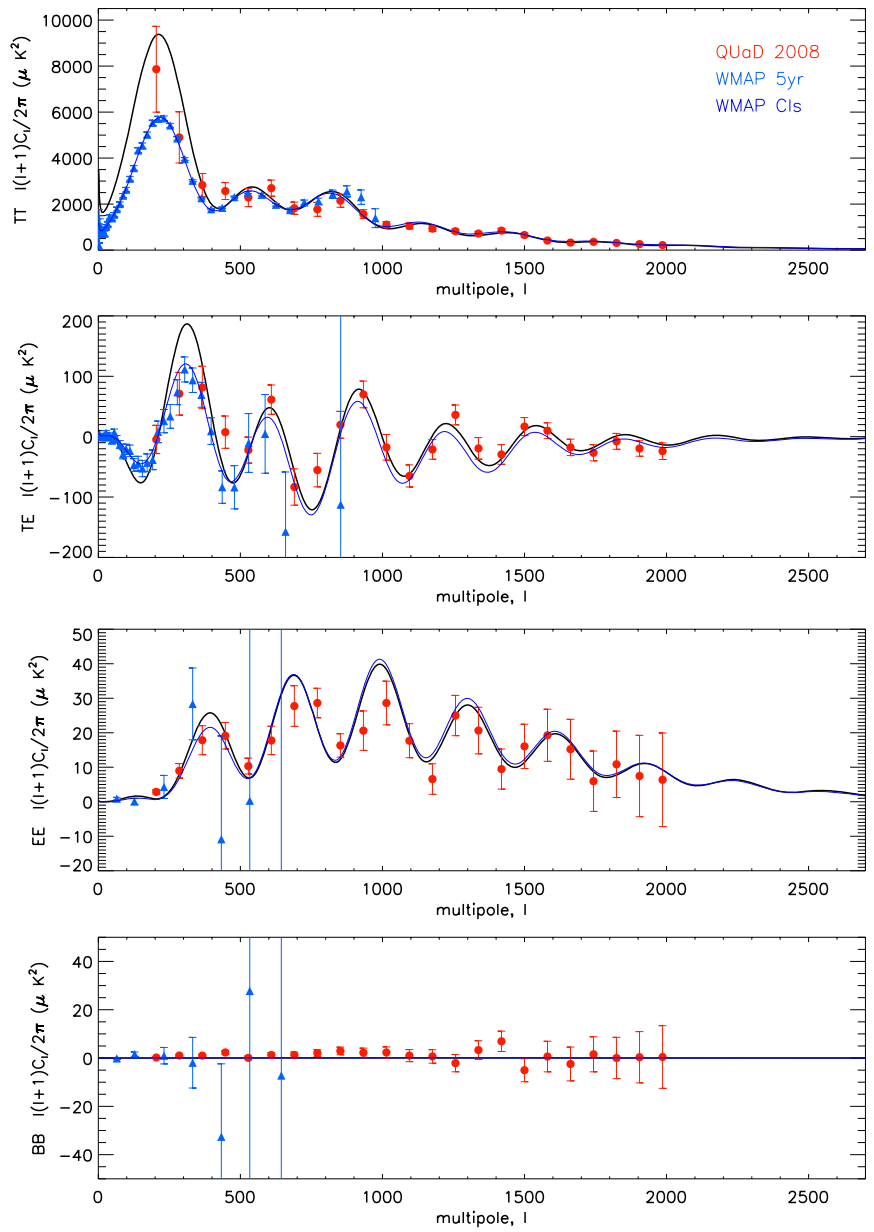

Figure 4. $\mathrm{QUaD}$ power spectra used in this analysis (red points) for $T T, T E$, $E E$, and $B B$ (top to bottom). The blue data points are $W M A P$ five-year power spectra data. The blue line shows the basic WMAP5 best-fit model as defined in Dunkley et al. (2009), while the black solid line shows our TT/TE/EE/BB mean recovered model with values given in Table 1 . We assumed the WMAP5 best-fit value for the optical depth $(\tau=0.087)$, which corresponds to an amplitude of $A_{s}=0.79$, given our $A_{s} e^{-2 \tau}$ constraint.

(A color version of this figure is available in the online journal.)

constraining them, have been an active field over the past few years (Kawasaki \& Sekiguchi 2007; Keskitalo et al. 2007; Bean et al. 2006; Beltran et al. 2004; Moodley et al. 2004 among many others). Pure isocurvature perturbations have been ruled out (Stompor et al. 1996; Langlois \& Riazuelo 2000; Enqvist et al. 2000; Amendola et al. 2002) although the presence of a subdominant isocurvature fraction has been claimed (Keskitalo et al. 2007). Observationally, isocurvature modes have a phase difference from adiabatic modes, which provides a distinct signature.

We can completely characterize the primordial perturbations by one adiabatic and several isocurvature components. The adiabatic component is given by the associated curvature perturbation $\mathcal{R}$ corresponding to an initial overdensity $\delta=\delta \rho / \rho$. The nonadiabatic components are given by entropy perturbations $\mathcal{S}_{x}=\delta_{x}-(3 / 4) \delta_{\gamma}$ between photons and a different species, $x$. These correspond to four possible nondecaying isocurvature modes: baryon density, cold dark matter density (cdmi), neutrino density (ndi), and neutrino velocity (nvi). Bucher et al. (2000) have presented a thorough analysis of these components.

We parameterize the contribution of adiabatic and isocurvature modes to the total temperature and polarization power 
Table 2

Basic Mean Parameters for QUaD TT/TE/EE/BB, SDSS LRG, and WMAP5 Data

\begin{tabular}{lccc}
\hline \hline Symbol & Q08 $T T / T E / E E / B B+$ WMAP5 & Q08 TT/TE/EE/BB+WMAP5+SDSS & WMAP5 \\
\hline$\Omega_{b} h^{2}$ & $0.02233_{-0.00057}^{+0.00055}$ & $0.02235_{-0.00058}^{+0.0051}$ & $0.02259_{-0.00062}^{+0.00061}$ \\
$\Omega_{m} h^{2}$ & $0.1266 \pm 0.0060$ & $0.1266_{-0.0038}^{+0.0039}$ & $0.1329 \pm 0.0065$ \\
$h$ & $0.733 \pm 0.027$ & $0.731 \pm 0.019$ & $0.715_{-0.027}^{+0.027}$ \\
$\tau$ & $0.087 \pm 0.017$ & $0.087 \pm 0.016$ & $0.087 \pm 0.017$ \\
$A_{s}{ }^{a}$ & $0.805 \pm 0.038$ & $0.806_{-0.033}^{+0.032}$ & $0.816 \pm 0.039$ \\
$n_{s}{ }^{a}$ & $0.960_{-0.013}^{+0.014}$ & $0.90_{-0.012}^{+0.014}$ & $0.966_{-0.015}^{+0.014}$ \\
\hline
\end{tabular}

Note.

a The pivot point for $A_{s}$ and $n_{s}$ is $k_{p}=0.002 \mathrm{Mpc}^{-1}$ for QUaD, WMAP5, and SDSS LRG data.

Table 3

CDM Isocurvature Mean Parameter Constraints for QUaD $T T / T E / E E / B B$, WMAP5, and SDSS LRG Data

\begin{tabular}{|c|c|c|c|}
\hline Symbol & Q08 $T T / T E / E E / B B+\mathrm{WMAP5}$ & Q08 TT/TE/EE/BB+WMAP5+SDSS & WMAP5 \\
\hline$\Omega_{b} h^{2}$ & $0.02312_{-0.00081}^{+0.00080}$ & $0.02280 \pm 0.00070$ & $0.02362_{-0.00094}^{+0.00096}$ \\
\hline$\Omega_{m} h^{2}$ & $0.1214_{-0.0068}^{+0.0067}$ & $0.1256_{-0.0037}^{+0.0038}$ & $0.1279 \stackrel{+0.0072}{-0.0071}$ \\
\hline$h$ & $0.773 \pm 0.039$ & $0.746 \pm 0.022$ & $0.759 \pm 0.041$ \\
\hline$\tau$ & $0.087 \pm 0.017$ & $0.084{ }_{-0.016}^{+0.016}$ & $0.087 \pm 0.017$ \\
\hline$A_{s}{ }^{\mathrm{a}}$ & $0.786 \pm 0.037$ & $0.797_{-0.030}^{+0.031}$ & $0.789 \pm 0.038$ \\
\hline$n_{s}^{\mathrm{a}}$ & $0.987 \pm 0.023$ & $0.976_{-0.017}^{+0.018}$ & $0.998_{-0.026}^{+0.027}$ \\
\hline$\alpha_{\text {cdmi }}(95 \%$ CL $)$ & $<0.19$ & $<0.11$ & $<0.21$ \\
\hline
\end{tabular}

Note.

a The pivot point for $A_{s}$ and $n_{s}$ is $k_{p}=0.002 \mathrm{Mpc}^{-1}$ for QUaD, WMAP, and SDSS data.

Table 4

NDI Isocurvature Mean Parameter Constraints for QUaD $T T / T E / E E / B B$, WMAP5, and SDSS LRG Data

\begin{tabular}{|c|c|c|c|}
\hline Symbol & Q08 TT/TE/EE/BB+WMAP5 & Q08 $T T / T E / E E / B B+\mathrm{WMAP} 5+\mathrm{SDSS}$ & WMAP5 \\
\hline$\Omega_{b} h^{2}$ & $0.02370 \pm 0.00110$ & $0.02300 \pm 0.00080$ & $0.02410_{-0.00120}^{+0.00130}$ \\
\hline$\Omega_{m} h^{2}$ & $0.1200_{-0.0072}^{+0.0073}$ & $0.1261 \pm 0.0039$ & $0.1270_{-0.0077}^{+0.0075}$ \\
\hline$h$ & $0.800 \pm 0.030$ & $0.751{ }_{-0.025}^{+0.027}$ & $0.780_{-0.055}^{+0.058}$ \\
\hline$\tau$ & $0.090 \pm 0.017$ & $0.085 \pm 0.016$ & $0.090 \pm 0.017$ \\
\hline$A_{s}{ }^{\mathrm{a}}$ & $0.869 \pm 0.058$ & $0.855_{-0.051}^{+0.053}$ & $0.872 \pm 0.059$ \\
\hline$n_{s}{ }^{\mathrm{a}}$ & $0.995_{-0.0252}^{+0.0271}$ & $0.976_{-0.017}^{+0.020}$ & $1.003_{-0.030}^{+0.031}$ \\
\hline$\alpha_{\text {ndi }}(95 \% \mathrm{CL})$ & $<0.37$ & $<0.26$ & $<0.38$ \\
\hline
\end{tabular}

Note.

a The pivot point for $A_{s}$ and $n_{s}$ is $k_{p}=0.002 \mathrm{Mpc}^{-1}$ for QUaD, WMAP, and SDSS data.

spectra by

$$
C_{\ell}^{X}=A_{s}^{2}\left[(1-\alpha) \hat{C}_{\ell}^{X, \mathrm{Ad}}+\alpha \hat{C}_{\ell}^{X, \text { Iso }}\right],
$$

where $\alpha$ is the isocurvature fraction. The adiabatic spectra, $C_{\ell}^{X, \text { Ad }}$, and the isocurvature spectra, $C_{\ell}^{X \text {,Iso }}$, are defined with unit amplitude and the same spectral index. In this analysis, we shall assume that there is no correlation between adiabatic and isocurvature modes, and will constrain one isocurvature mode at a time. Also we do not present results for the baryon density isocurvature mode as these have a very similar signature to the cold dark matter mode.

We analyze the QUaD $T T / T E / E E / B B$ power spectra combined with WMAP5, and combined with the WMAP5 plus the SDSS LRG data. The shape of the galaxy power spectrum is sensitive to an isocurvature contribution, and has been used in the past to improve on isocurvature constraints (e.g., Beltran et al. 2004, 2005; Crotty et al. 2003). The results we obtain are given in Table 3 for the cdmi mode, in Table 4 for the ndi mode, and in Table 5 for the nvi mode.

Our analysis shows a small improvement in the isocurvature cold dark matter constraint when we add the QUaD to the WMAP5 data, from $\alpha_{\text {cdmi }}<0.21$ to $\alpha_{\text {cdmi }}<0.19(95 \%$ confidence limits (CLs)). In addition, we find an improvement in the $\Omega_{b} h^{2}$ and $\Omega_{m} h^{2}$ constraints. There is a similar improvement for the neutrino density isocurvature constraints: we go from $\alpha_{\text {ndi }}<0.38$ to $\alpha_{\text {ndi }}<0.37$. For the neutrino velocity isocurvature modes, there is no improvement, the constraint staying at $\alpha_{\text {nvi }}<0.27$.

We can further improve on these results by adding the SDSS LRG data. When we do this, the cold dark matter isocurvature constraint becomes $\alpha_{\mathrm{cdmi}}<0.11$ (95\% CL). The largest improvement is for the neutrino density isocurvature mode, $\alpha_{\text {ndi }}<0.26$, while the smallest improvement is for the neutrino velocity isocurvature mode, $\alpha_{\text {nvi }}<0.23$.

\section{SUMMARY AND CONCLUSIONS}

We have presented a standard cosmological parameter constraint analysis, and its extension to include isocurvature modes, using QUaD TT, TE, EE, and $B B$ band-power spectra. This is the first CMB experiment to detect with confidence the acoustic oscillations in the $E E$ spectrum and so the first to be able to provide significant constraints on cosmological parameters from individual CMB polarization spectra-in fact our polarization-only constraints are superior to our temperature-only constraints. 


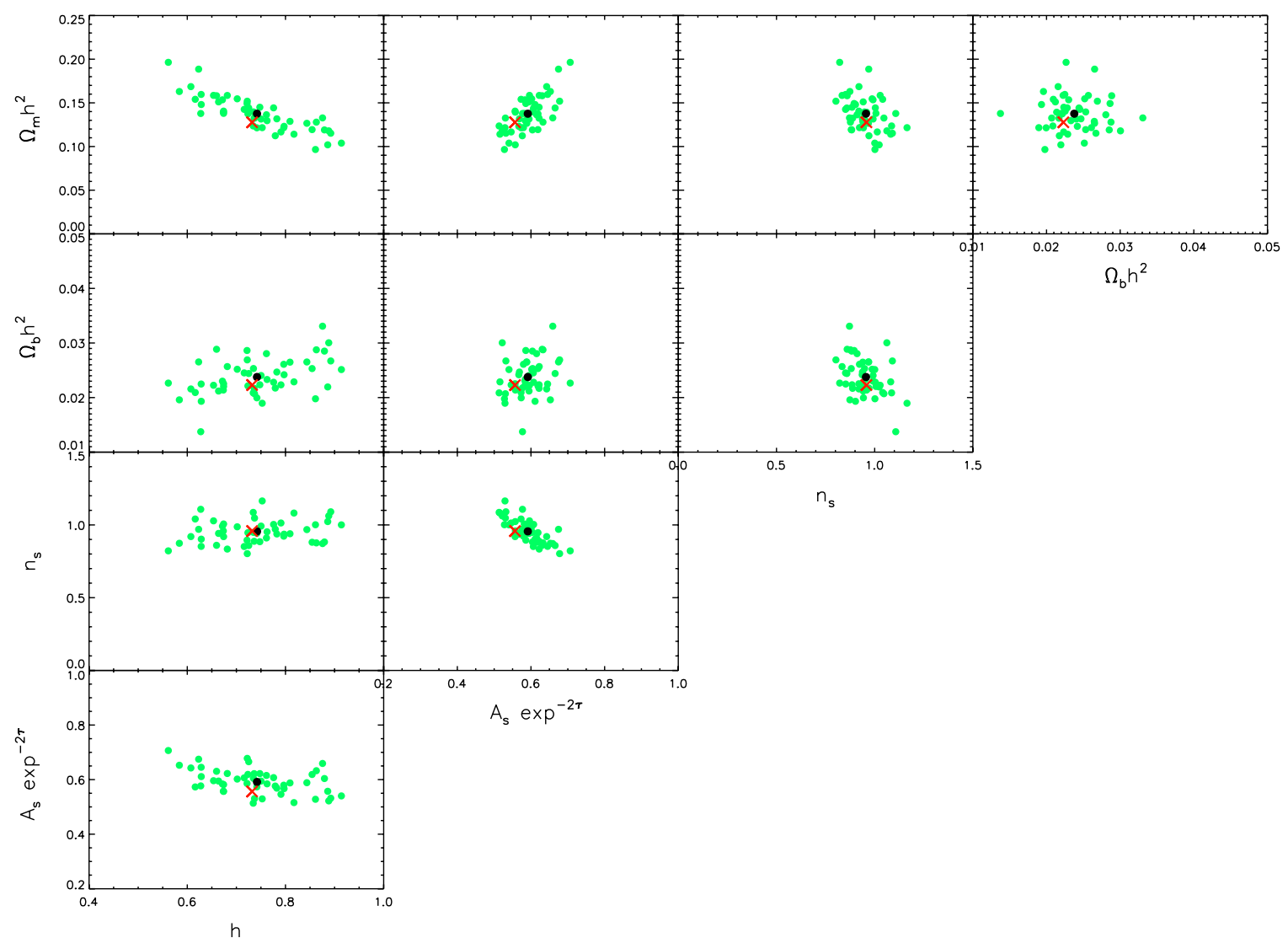

Figure 5. Two-dimensional marginalized plot, showing the scattered values (green) of the five parameters mean recovered basic cosmological model obtained from 50 simulations of QUaD TT, TE, EE, and $B B$ data generated from the WMAP3 best-fit model (in red) from Table 2 of Spergel et al. (2007). The mean over the 50 simulations is shown as a blue point.

(A color version of this figure is available in the online journal.)

Table 5

NVI Isocurvature Mean Parameter Constraints for QUaD TT/TE/EE/BB, WMAP5, and SDSS LRG Data

\begin{tabular}{lccc}
\hline \multicolumn{1}{c}{ Symbol } & Q08 TT/TE/EE/BB+WMAP5 & Q08 TT/TE/EE/BB+WMAP5+SDSS & WMAP5 \\
\hline$\Omega_{b} h^{2}$ & $0.02350 \pm 0.00090$ & $0.02339_{-0.00070}^{+0.00080}$ & $0.02390 \pm 0.00100$ \\
$\Omega_{m} h^{2}$ & $0.1260 \pm 0.0060$ & $0.1277 \pm 0.0040$ & $0.1330 \pm 0.0063$ \\
$h$ & $0.745 \pm 0.029$ & $0.734^{2} \pm 0.018$ & $0.728_{-0.028}^{+0.027}$ \\
$\tau$ & $0.088^{2} \pm 0.017$ & $0.087_{-0.015}^{+0.016}$ & $0.089 \pm 0.018$ \\
$A_{s}{ }^{a}$ & $0.851_{-0.047}^{+0.048}$ & $0.854_{-0.045}^{+0.044}$ & $0.859 \pm 0.048$ \\
$n_{s}{ }^{\mathrm{a}}$ & $0.980_{-0.018}^{+0.017}$ & $0.978_{-0.014}^{+0.015}$ & $0.988_{-0.018}^{+0.019}$ \\
$\alpha_{\text {nvi }}(95 \% \mathrm{CL})$ & $<0.27$ & $<0.23$ & $<0.27$ \\
\hline
\end{tabular}

Note.

a The pivot point for $A_{s}$ and $n_{s}$ is $k_{p}=0.002 \mathrm{Mpc}^{-1}$ for QUaD, WMAP, and SDSS data.

In combination with the WMAP5 data set, QUaD offers a small improvement in the constraints on the baryon and matter densities.

We find that our QUaD temperature data are in good agreement with the results from WMAP5, which is a nontrivial test of $\Lambda \mathrm{CDM}$ as the QUaD data extends to $\ell \approx 2000$ with good signal to noise. However, our polarization (TE, EE, and $B B$ ) data are in less good agreement yielding a higher baryon density value of $\Omega_{b} h^{2}=0.0319 \pm 0.0046$, compared with $\Omega_{b} h^{2}=0.0242_{-0.0057}^{+0.0058}$ from our TT data and $0.02261_{-0.00063}^{+0.00062}$ from our reanalysis of WMAP5. A $\chi^{2}$ test shows that there is a 7\% probability of the $\mathrm{QUaD}$ polarization results arising by chance, assuming the WMAP5 $\Lambda$ CDM model is correct. Although not of high significance, this modest level of tension, that seems to originate from the $T E$ spectrum, could be due to new physics in polarization, residual systematics in the data, or random chance. It will be interesting to see if this trend continues in future polarization experiments.

We also investigate isocurvature cold dark matter density, neutrino density and neutrino velocity modes. We find that $\mathrm{QUaD}$ provides a marginal improvement on the fractional cold dark matter density mode parameter, $\alpha_{\mathrm{cdmi}}$, from $<0.21$ for WMAP5 alone to $<0.19$.

QUaD is funded by the National Science Foundation in the USA, through grants AST-0096778, ANT-0338138, ANT-0338335, and ANT-0338238, by the UK Science and Technology Facilities Council (STFC) and its predecessor the Particle Physics and Astronomy Research Council (PPARC), and by the Science Foundation Ireland. J.R.H. acknowledges 
the support of an NSF Graduate Research Fellowship, a Stanford Graduate Fellowship and a NASA Postdoctoral Fellowship. C.P. and J.E.C. acknowledge partial support from the Kavli Institute for Cosmological Physics through the grant NSF PHY-0114422. E.Y.W. acknowledges receipt of an NDSEG fellowship. Y.M. acknowledges support from a SUPA Prize studentship. P.G.C. acknowledges funding from the Fundação para a Ciência e a Tecnologia. M.Z. acknowledges support from a NASA Postdoctoral Fellowship. This work has made use of the resources provided by the Edinburgh Compute and Data Facility (ECDF) that is partially supported by the eDIKT initiative. We also thank Licia Verde and Joanna Dunkley for useful discussion.

\section{APPENDIX}

\section{SIMULATING PARAMETER ESTIMATION}

We test our MCMC pipeline by running it on a set of 50 simulations of the QUaD $T T, T E, E E$, and $B B$ band-power spectra. These were generated by simulating the signal and noise properties of the time-ordered data and passing these through the QUaD pipeline in the same way as the data (see the Power Spectra Paper for details). The input cosmological model for these simulations was the WMAP3 mean recovered model (see Table 2 of Spergel et al. 2007). The scatter in the values of the mean recovered model obtained from each one of the 50 simulations can be seen in Figure 5. We also overplot the average values for each parameter calculated from the 50 simulations (see blue points). We have verified that the scatter in the simulated mean parameter results is close to the size of the contours produced by our MCMC code when using real data, indicating that our code accurately estimates the parameter uncertainties.

We can also compare the mean parameter values and the scatter about them with the input WMAP3 best-fit model (red crosses). The average over the simulations closely matches the input model indicating that our parameters are not biased. If constrained independently, the scalar amplitude, $A_{s}$, and the optical depth, $\tau$, parameters are biased, their values being systematically higher than the input values. This is due to the combination of the large degeneracy between the amplitude, $A_{s}$, and optical depth, $\tau$, and the parameter priors. To break this degeneracy requires large-scale polarization measurements probing the reionization bumps at lower $\ell$-modes. As can be seen in the figure, this problem can be avoided if we combine $A_{s}$ and $\tau$ into the parameter $A_{s} e^{-2 \tau}$ along the line of degeneracy, which is the approach followed in Section 3.1.

\section{REFERENCES}

Ade, P., et al. 2008, ApJ, 674, 22

Amendola, L., Gordon, C., Wands, D., \& Sasaki, M. 2002, Phys. Rev. Lett., 88, 211302

Barkats, D., et al. 2005, ApJ, 619, L127

Bean, R., Dunkley, J., \& Pierpaoli, E. 2006, Phys. Rev. D, 74, 063503

Beltran, M., Garcia-Bellido, J., Lesgourgues, J., \& Riazuelo, A. 2004, Phys. Rev. D, 70, 103530

Beltran, M., Garcia-Bellido, J., Lesgourgues, J., \& Viel, M. 2005, Phys. Rev. D, 72, 103515

Bischoff, C., et al. 2008, ApJ, 684, 771

Bond, J. R., Jaffe, A. H., \& Knox, L. 1998, Phys. Rev. D, 57, 2117

Bridle, S. L., Crittenden, R., Melchiorri, A., Hobson, M. P., Kneissl, R., \& Lasenby, A. N. 2002, MNRAS, 335, 1193

Brown, M. L., Castro, P. G., \& Taylor, A. N. 2005, MNRAS, 360, 1262

Bucher, M., Moodley, K., \& Turok, N. 2000, Phys. Rev. D, 62, 083508

Crotty, P., Garcia-Bellido, J., Lesgourgues, J., \& Riazuelo, A. 2003, Phys. Rev. Lett., 91, 171301

Dunkley, J., et al. 2009, ApJS, 180, 306

Enqvist, K., Kurki-Suonio, H., \& Valiviita, J. 2000, Phys. Rev. D, 62, 103003

Gelman, A., \& Rubin, D. B. 1992, Statist. Sci., 7, 457

Hu, W., \& White, M. J. 1997, Phys. Rev. D, 56, 596

Kawasaki, M., \& Sekiguchi, T. 2007, arXiv:0705.2853

Keskitalo, R., Kurki-Suonio, H., Muhonen, V., \& Valiviita, J. 2007, J. Cosmol. Astropart. Phys., JCAP09(2007)008

Kirkman, D., Tytler, D., Suzuki, N., O’Meara, J. M., \& Lubin, D. 2003, ApJS, 149,1

Kosowsky, A., Milosavljevic, M., \& Jimenez, R. 2002, Phys. Rev. D, 66, 063007

Kovac, J. M., Leitch, E. M., Pryke, C., Carlstrom, J. E., Halverson, N. W., \& Holzapfel, W. L. 2002, Nature, 420, 772

Langlois, D., \& Riazuelo, A. 2000, Phys. Rev. D, 62, 043504

Lewis, A., Challinor, A., \& Lasenby, A. 2000, ApJ, 538, 473

Metropolis, N., Rosenbluth, A., Rosenbluth, M. N., Teller, A. H., \& Teller, E. 1953, J. Chem. Phys., 21, 1087

Montroy, T. E., et al. 2006, ApJ, 647, 813

Moodley, K., Bucher, M., Dunkley, J., Ferreira, P. G., \& Skordis, C. 2004, Phys. Rev. D, 70, 103520

Nolta, M. R., et al. 2009, ApJS, 180, 296

Page, L., et al. 2003, ApJS, 148, 233

Pryke, C., et al. 2009, ApJ, 692, 1247

Readhead, A. C. S., et al. 2004, Science, 306, 836

Reichardt, C. L., et al. 2009, ApJ, 694, 1200

Sievers, J. L., et al. 2007, ApJ, 660, 976

Spergel, D. N., et al. 2007, ApJS, 170, 377

Stompor, R., Banday, A. J., \& Gorski, Krzysztof, M. 1996, ApJ, 463, 8

Tegmark, M., et al. 2004, Phys. Rev. D, 69, 103501

Tegmark, M., et al. 2006, Phys. Rev. D, 74, 123507

Verde, L., et al. 2003, ApJS, 148, 195 